\title{
Cigarette Smoke Mediates Nuclear to Cytoplasmic Trafficking of Transcriptional Inhibitor Kaiso through MUC1 and P120-Catenin
}

\author{
Lili Zhang, ${ }^{*}$ Marianne Gallup, ${ }^{*}$ Lorna Zlock, ${ }^{\dagger}$ Yu Ting Feeling Chen, ${ }^{*}$ Walter E. Finkbeiner, ${ }^{\dagger}$ and Nancy A. McNamara ${ }^{\star \delta}$
}

From the Francis I. Proctor Foundation* and the Departments of Pathology ${ }^{\dagger}$ and Anatomy and Ophthalmology, ${ }^{\ddagger}$ University of California, San Francisco, San Francisco; and the School of Optometry and Vision Science Graduate Program, ${ }^{\S}$ University of California, Berkeley, Berkeley, California

Accepted for publication

August 29, 2016.

Address correspondence to Nancy A. McNamara, Ph.D., O.D., 513 Parnassus Ave., Box 0412, San Francisco, CA 94143. E-mail: nancy. mcnamara@ucsf.edu.

\begin{abstract}
Lung cancer is the leading cause of cancer-related death, and $87 \%$ of these deaths are directly attributable to smoking. Using three-dimensional cultures of primary human bronchial epithelial cells, we demonstrated that loss of adherens junction protein, epithelial cadherin, and the aberrant interaction of its adherens junction binding partner, p120-catenin (p120ctn), with the cytoplasmic tail of apical mucin-1 (MUC1-CT) represent initiating steps in the epithelial-to-mesenchymal transition. Smoke provoked the rapid nuclear entry of p120ctn in complex with MUC1-CT that was inhibited using the MUC1-CT inhibitory peptides, PMIP and G0-201. Nuclear entry of p120ctn promoted its interaction with transcriptional repressor kaiso and the rapid shuttling of kaiso to the cytoplasm. Nuclear exit of kaiso permitted the up-regulation of oncogenic transcription factors Fos/phospho-Ser ${ }^{32}$ Fos, FosB, Fra1/ phospho-Ser ${ }^{265}$ Fra1, which was inhibited through suppression of p120ctn's nuclear export using leptomycin-B. These data indicated that smoke-induced nuclear-to-cytoplasmic translocation of kaiso depends on the nuclear import of p120ctn in complex with MUC1-CT and the nuclear export of kaiso in complex with p120ctn. The presence of MUC1-CT/p120ctn and p120ctn/kaiso complexes in lung squamous cell carcinoma and adenocarcinoma specimens from human patients confirms the clinical relevance of these events. Thus, enhancing kaiso's suppressor role of protumor genes by sequestering kaiso in the nucleus of a smoker's airway epithelium may represent a novel approach of treating lung cancer. (Am J Pathol 2016, 186: 3146-3159; http://dx.doi.org/10.1016/j.ajpath.2016.08.011)
\end{abstract}

Cigarette smoke increases the risk of developing lung cancer by 20 -fold and is responsible for $87 \%$ of lung cancer deaths. In response to the overwhelming need for effective therapies to fight smoking-related deaths, we are working to gain a better understanding of molecular events leading to lung carcinogenesis in the smoker's airway. ${ }^{1-5}$

Mucin-1 (MUC1) is a heavily, O-glycosylated, transmembrane glycoprotein expressed on the apical surface of mucosal epithelia in the lung, eye, breast, and stomach. ${ }^{6}$ In the normal airway, mucins and adherens junctions (AJs) establish a structural barrier that protects the airway from infectious, inflammatory, and noxious stimuli. It is well documented by our laboratory and others that smoke disrupts cell-cell junctions via its damaging effects on the AJ protein, epithelial cadherin (E-cad). ${ }^{3,4}$ Loss of E-cad is a major hallmark of epithelial-mesenchymal transition (EMT) and has been reported in lung squamous cell carcinoma (SCC) and adenocarcinoma (ADCA) where it is associated with invasion, metastasis, and poor prognosis.

With the use of organotypic cultures of primary human bronchial epithelial (HBE) cells, we demonstrated that Ecad loss is regulated through the aberrant interaction of its AJ binding partner, p120-catenin (p120ctn), and the cytoplasmic (CYTO) tail of MUC1 (MUC1-CT). ${ }^{4}$ Accordingly, blocking MUC1-CT's interaction with p120ctn using the MUC1 inhibitory peptide, PMIP, prevented E-cad loss in response to smoke. ${ }^{4}$ Moreover, we have shown that the

Supported by American Cancer Society grant 115502-RSG-08-136-01CNE, NIH grant DK72517, and a Research and Development grant from the Cystic Fibrosis Foundation.

Disclosures: None declared. 
smoke-induced interaction between MUC1-CT and p120ctn is preceded by aberrant glycosylation of MUC1's N-terminus. Inhibition of smoke-induced glycosylation of MUC1's $\mathrm{N}$-terminus using adenoviral siRNA directed against $\mathrm{N}$-acetyl-galactosaminyl transferase-6, an enzyme that controls the initiation step of O-linked glycosylation, led to reduced MUC1-CT/p120ctn complex formation and prevented E-cad degradation in response to smoke. ${ }^{5}$ These data provide evidence that MUC1-CT/p120ctn interaction induced by cigarette smoke represents an initiating step of EMT, during which airway epithelial cells are transformed into highly motile mesenchymal cells due to disruption of AJ gatekeeper, E-cad. ${ }^{4,5}$ With the molecular events underlying smoke-induced EMT critical for lung cancer progression, we believe that blocking EMT through the disruption of MUC1-CT/p120ctn complex formation and stabilization of the AJs represents a promising method to treat lung cancer in its premalignant stages. ${ }^{8}$

Kaiso belongs to the BTB/POZ (Broad complex, Tramtrak, bric à brac/poxvirus and zinc finger family) of zinc finger (POZ-ZF) proteins that was originally identified as a binding partner of p120ctn through yeast two-hybrid screen. ${ }^{9}$ Different from other POZ-ZF proteins, kaiso displays bimodal DNA-binding capacity; it associates with a sequence-specific DNA consensus kaiso binding site (TCCTGCnA) or adjoining methylated $\mathrm{CpG}$ dinucleotides (CpG islands) in various promoters. ${ }^{10}$ Kaiso binds DNA directly using two of three adjacent zinc finger motifs near its C-terminus, ${ }^{10}$ whereas its $\mathrm{N}$-terminal POZ domain facilitates kaiso homodimerization and recruits transcription co-repressor such as nuclear receptor co-repressor $1 .{ }^{11}$ Through its interaction with kaiso, p120ctn modulates noncanonical Wnt gene targets such as Wnt11, which signals through Rho GTPase and plays a prominent role in morphogenesis and cell adhesion. ${ }^{12}$ Along with $\mathrm{T}$ cell factor/ $\beta$-catenin $(\beta$-ctn) complexes, p120ctn coordinately regulates canonical Wnt gene targets by kaiso, such as matrix metalloprotease Matrilysin, cyclin-D1, Siamois, c-Fos, and c-Myc. ${ }^{10,13}$ So far, p120ctn's involvement in regulation of gene transcription has mostly been seen when kaiso is associated with the kaiso binding site. ${ }^{14}$ It has recently been reported that p120ctn can regulate $\beta$-ctn transcription through kaiso's association to the $\mathrm{CpG}$ islands in its promoter in lung cancer cells. ${ }^{15}$ This is particularly intriguing because it indicates kaiso's role in the regulation of some Wnt targets via binding to $\mathrm{CpG}$ islands, an extensively studied hallmark of tumor suppressor silencing.

It is unknown whether p120ctn is involved in the regulation of transcription of a subset of protumor genes through its interaction with kaiso after smoke exposure. Here, we used the model system of pseudostratified, polarized, primary HBE cells to mimic the in vivo airway. ${ }^{3-5} \mathrm{We}$ revealed that smoke-induced MUC1-CT/p120ctn interaction facilitated the nuclear (NUC) translocation of p120ctn where it interacted with the transcriptional repressor, kaiso. Smoke-provoked kaiso/p120ctn interaction assisted NUC-
to-CYTO translocation of kaiso, thus releasing kaiso from its repressor function, and thereby permitting the activation of c-Fos transcription factors.

\section{Materials and Methods}

\section{Culture and Smoke Treatment of Pseudostratified HBE Cells}

Pseudostratified HBE cell cultures were established and treated by smoke-concentrated medium (Smk) as we described previously. ${ }^{4,5}$

\section{Lung Carcinoma Tissue Blocks}

Formalin-fixed, paraffin-embedded human lung SCC and ADCA tissue sections specimens (51- to 66-year-old, all men) were obtained from the San Francisco General Hospital Department of Anatomical Pathology. The images shown are representative for a few patients. The smoking history of lung cancer patients is unknown due to the Health Insurance Portability and Accountability Act violation to go into the patient's medical record. Lung carcinoma tissues were fixed with $10 \%$ neutral buffered formalin, processed in an automated tissue processor (Tissue-Tek VIP; Sakura Finetek, Torrance, CA) and embedded in paraffin. Paraffin microscopic sections (5 $\mu \mathrm{m})$ were de-waxed in xylene and rehydrated through a graduated alcohol series and water before use.

\section{Human Research Approval}

All protocols involving the use of human tissues, cells, and tissue sections were reviewed and approved by the University of California San Francisco Committee on Human Research. Written consent was not required because materials were leftover clinical samples obtained from deidentified individuals.

\section{Reagents and Plasmid Constructs}

Human MUC1 inhibitory peptide PMIP ( $\mathrm{NH}^{-}$-YARAAARQARAPYEKVSAGNGGSSLS- $\left.{ }^{-}{ }{ }^{16}\right)^{16}$ was synthesized by GenScript (Piscataway, NJ). Human MUC1 inhibitory peptide GO-201 (GO; NH2-[R] $]_{9}$ CQCRRKNYGQLDIFP- $\mathrm{COOH}$ ) and the inhibitor of protein NUC export leptomycin-B (LMB) were purchased from Sigma-Aldrich (St. Louis, MO).

MUC1 cDNA (NM_002456.4) was purchased from OriGene Technologies Inc. (Rockville, MD). MUC1-CT was amplified from MUC1 cDNA with primers modified from a previous publication ${ }^{17}$ [forward primer with XhoI site: 5'-ACCTCGAGATCGTAATGATACAATTGACTCTGGCCTTC- $3^{\prime}$ (underline shows the XhoI site); reverse primer with EcoRI site: 5'-AGGAATTCGCAAGTTGGCAGAAGTGGCTG-3' (underline shows the EcoRI site)]. 
A

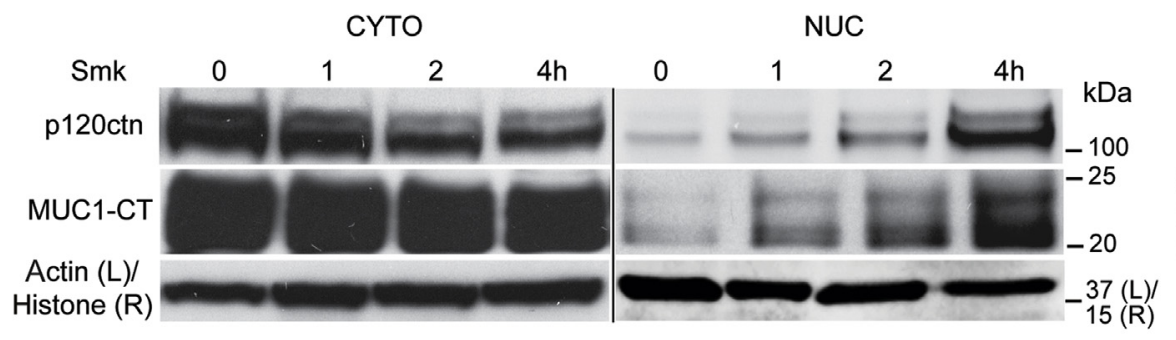

C

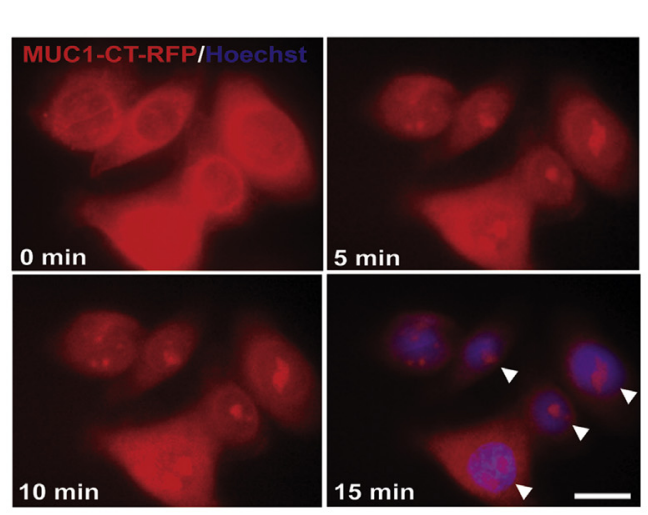

D
B

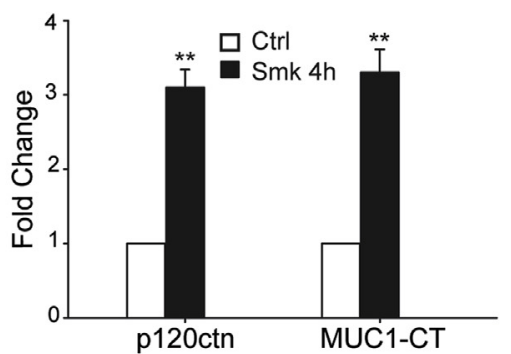

Ctrl $4 \mathrm{~h}$

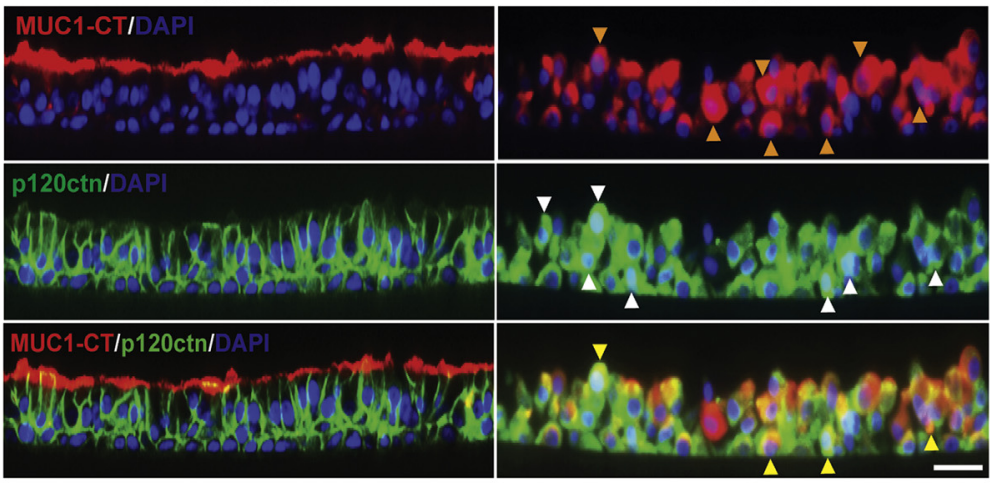

E

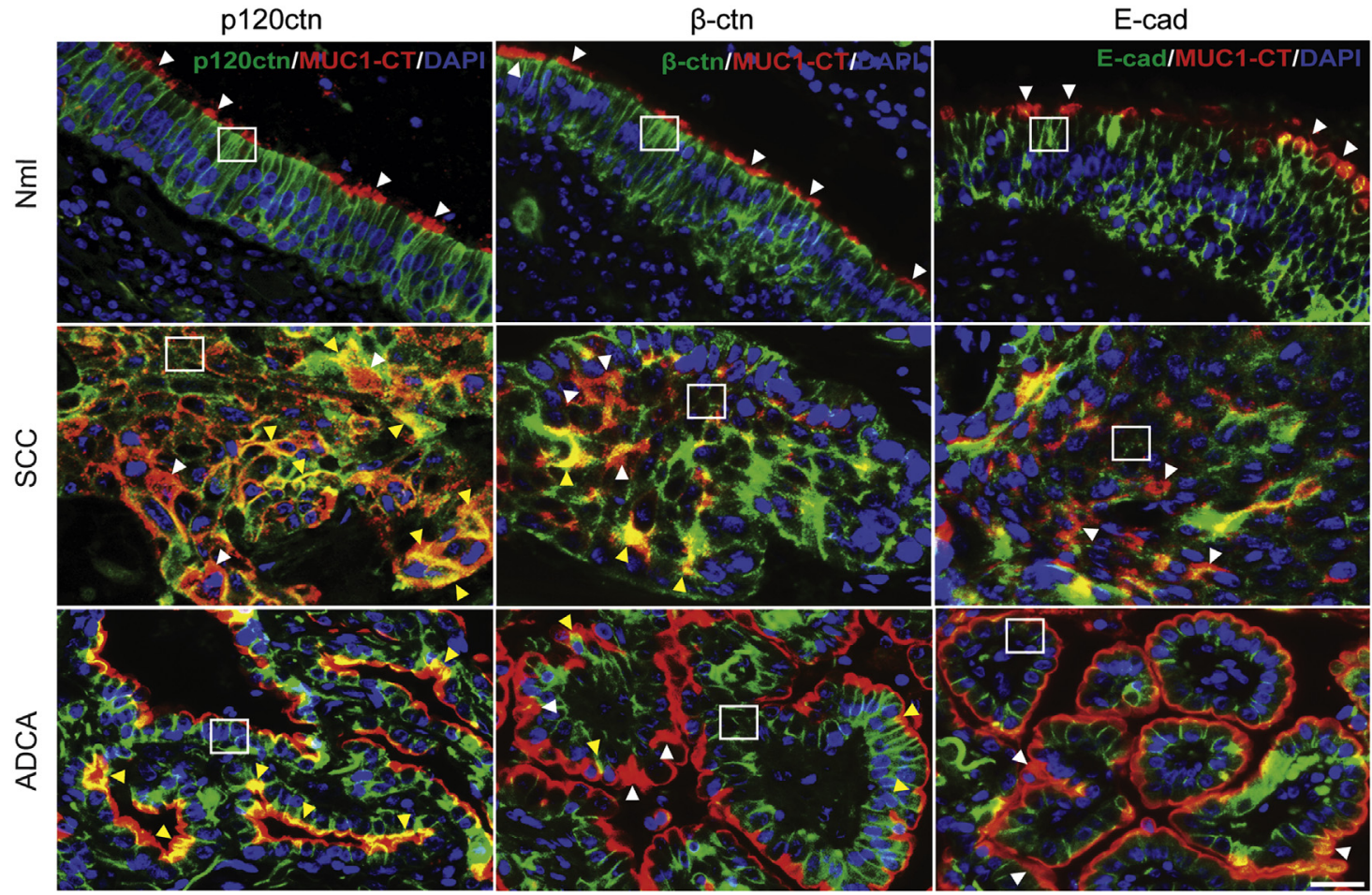


The MUC1-CT product was sequenced and cloned between the XhoI and EcoRI sites of pRFP-N1 (a gift from Dr. Brigitte E. Blackman, University of California, San Francisco) to make MUC1-CT-RFP (red fluorescent protein) with RFP tag fused to the C-terminus of MUC1-CT. A kaiso-green fluorescent protein (GFP) construct with GFP fused to N-terminus of kaiso was obtained from Dr. PierreAntoine Defossez (Institut Curie, Paris, France). ${ }^{18}$ The plasmids were transfected into primary $\mathrm{HBE}$ cells using Amaxa nucleofector normal HBE kit (Lonza Ltd., Basel, Switzerland) according to manufacturer's instructions. Experiments were generally performed at 48 hours after transfection.

\section{Cell Fractionation, Immunoprecipitation, and WB Analysis}

NUC and CYTO fractions of cell lysates were extracted using NE-PER Nuclear and Cytoplasmic Extraction Kit (Thermo Fisher Scientific Inc., Waltham, MA) according to manufacturer's instructions. All samples were processed simultaneously by the same technician to achieve the same ratio of protein extraction for each sample. The extracted NUC and CYTO fractions were inspected with Western blot (WB) analysis probed with actin and histone antibodies. Only histone $^{+} /$actin $^{-}$NUC fractions and histone ${ }^{-} /$actin $^{+}$CYTO fractions were used for immunoprecipitation/WB analysis as previously described. ${ }^{4,5}$ Protein concentrations of the obtained samples were determined by a bicinchoninic acid protein assay kit (Thermo Scientific Pierce). Cell lysates $(200 \mu \mathrm{g})$ per sample was used for immunoprecipitation, and $30 \mu \mathrm{g}$ of cell lysates per sample was used for WB analysis. p120ctn, $\beta$-ctn, and Ecad antibodies were purchased from BD Transduction Laboratories (San Jose, CA). MUC1-CT2 antibody was obtained from Dr. Sandra J. Gendler (Mayo Clinic College of Medicine, Phoenix, AZ). ${ }^{19}$ Kaiso antibody (6F8) was purchased from Abcam (Cambridge, MA). p44/42 mitogen-activated protein kinase (Erk), phospho-Thr ${ }^{202} /$ Tyr $^{204}$ p44/42 MAPK (Erk-P), Fos, phospho-Ser ${ }^{32}$ Fos (Fos-P), Fra1, phospho-Ser ${ }^{265}$ Fra1
(Fra1-P), FosB, $\beta$-actin, and histone-H3 antibodies were obtained from Cell Signaling Technology Inc. (Danvers, MA). Densitometric quantification of bands was conducted using ImageJ software version $1.49 \mathrm{v}(\mathrm{NIH}$, Bethesda, MD).

\section{Immunofluorescent Staining}

Immunofluorescent staining was done as we previously described $^{4}$ using the p120ctn, $\beta$-ctn, and E-cad antibodies mentioned above. Kaiso antibody (NBP1-87866) was purchased from Novus Biologicals (Littleton, CO). Images were obtained with a Nikon-Eclipse Ti fluorescence microscope (Nikon, Tokyo, Japan) using NIS-elements AR software version 3.07.

\section{Real-Time Quantitative PCR}

Real-time quantitative PCR was done as we previously described. ${ }^{3}$ Total RNA was extracted from primary HBE cells using an RNeasy Mini RNA isolation kit (Qiagen, Piscataway, NJ). The cDNA was synthesized from $1 \mu \mathrm{g}$ of total RNA using ABI high-capacity cDNA reverse transcription kit (Applied Biosystems, Foster City, CA). Real-time quantitative PCR was performed using ABI SYBR Green PCR master mix with ABI Prism 7300 Real-Time PCR System (Applied Biosystems). The primer sequences of c-Fos, Fra1, glyceraldehyde-3-phosphate dehydrogenase, and FosB were obtained from previous publications. ${ }^{20-23}$ The data were collected and analyzed by the comparative threshold cycle method using glyceraldehyde-3-phosphate dehydrogenase expression as the reference gene. Data were collected in triplicate from three independent experiments.

\section{Statistical Analysis}

Three to four independent repeats were conducted in all experiments. Data are presented as means \pm SEM. A $t$-test was used, and a $P$ value of $<0.05$ was considered statistically significant.

\footnotetext{
Figure 1 Smoke induced time-dependent nuclear translocation of p120ctn and MUC1-CT. A: Polarized HBE cells were exposed to Smk for indicated time points. CYTO and NUC fractions were obtained and analyzed by Western blot analysis probed with p120ctn, MUC1-CT, actin (CYTO fractions; left panel) and histone (NUC fractions; right panel) antibodies. B: Densitometric analysis of p120ctn and MUC1-CT in NUC fractions after 4 hours of smoke exposure was normalized to those in untreated Ctrl (designated as onefold). C: MUC1-CT-RFP construct (red) was transfected into HBE cells by electroporation. At 48 hours after transfection, the cells were exposed to Smk for indicated time points. Cell nuclei were stained with Hoechst (blue, 15 minutes time point only). D: Pseudostratified HBE cells were exposed to Smk and Ctr for 4 hours. Immunofluorescent staining of HBE cells for p120ctn (green) and MUC1-CT (red) is shown. Cell nuclei were counterstained with DAPI (blue). Orange arrowheads point to localization of MUC1-CT in the nucleus (purple signals generated by overlying of $\mathrm{red} / \mathrm{blue}$ signals); white arrowheads, to localization of p120ctn in the nucleus (light blue signals generated by overlying of green/blue signals); yellow arrowheads, to colocalization of MUC1-CT and p120ctn in the nucleus (white signals generated by overlying of red/green/blue signals). E: Immunofluorescent staining of AJs in lung SCC, lung ADCA, and adjacent airway epithelium ( $\mathrm{Nml}$ ). Antibodies directed against p120ctn (green, left column), $\beta$-ctn (green, middle column), E-cad (green, right column), and MUC1-CT (red, all panels) are shown. Nuclei were visualized with DAPI (blue). White arrowheads point to MUC1-CT located at apical membrane in normal tissue (top row) versus cytoplasm in lung SCC and ADCA (middle and bottom rows). Yellow arrowheads point to MUC1$\mathrm{CT} / \mathrm{p} 120 \mathrm{ctn}$ and MUC1-CT/ $\beta$-ctn colocalization (yellow signals generated by overlying of red/green signals) at the intercellular junctions and cytoplasm in lung SCC/ADCA (middle and bottom rows). White boxes indicate AJs where p120ctn, $\beta$-ctn, and E-cad are intact in normal tissue (top row) but reduced in lung SCC and ADCA (middle and bottom rows). Data are expressed as means \pm SEM fold changes. ${ }^{*} P<0.01$. Scale bars: $20 \mu \mathrm{m}$ (C); $50 \mu \mathrm{m}$ (D and E). ADCA, adenocarcinoma; AJ, adherens junction; Ctrl, smoke-free medium; CYTO, cytoplasmic; E-cad, epithelial cadherin; HBE, human bronchial epithelial; L, left; MUC1-CT, cytoplasmic tail of mucin-1; Nml, normal; NUC, nuclear; p120ctn, p120-catenin; R, right; RFP, red fluorescent protein; SCC, squamous cell carcinoma; Smk, smoke-concentrated medium; $\beta$-ctn, $\beta$-catenin.
} 


\section{Results}

Smoke Induces Complex Formation of p120ctn and MUC1-CT in the Nuclei of Pseudostratified HBE Cells

We previously demonstrated the smoke-induced disruption of cell polarity and repositioning of MUC1-CT to the basolateral membrane of pseudostratified HBE cells where it interacted with p120ctn and provoked the degradation of E-cad. ${ }^{4}$ Here, we provide evidence to support the time-dependent NUC translocation of MUC1-CT and p120ctn after a 4-hour exposure to smoke. Compared with pseudostratified HBE cells grown in basal medium, smoke increased NUC levels of MUC1-CT by 3.4-fold in conjunction with a 3.1-fold increase in NUC p120ctn (Figure 1, A and B). Consistent with this finding, MUC1-CT-RFP was localized in the cytoplasm of HBE cells but relocated into the nuclei within 5 minutes of smoke exposure. Simultaneous Hoechst staining confirmed accumulation of MUC1-CT in the nucleus of HBE cells through 15 minutes of smoke exposure (Figure 1C). Immunostaining with MUC1-CT and p120ctn antibodies revealed relocation of apical MUC1-CT and junctional p120ctn in the nucleus as well as colocalization of MUC1-CT/p120ctn in the nucleus of pseudostratified HBE cells after 4 hours of smoke exposure (Figure 1D). The NUC localization of p120ctn suggests its potential role in the regulation of gene transcription in smoke-exposed airway epithelial cells.

The staining pattern in lung cancer specimens closely mimicked that of the fully differentiated, polarized, primary HBE cells used for our studies. In the human airway,
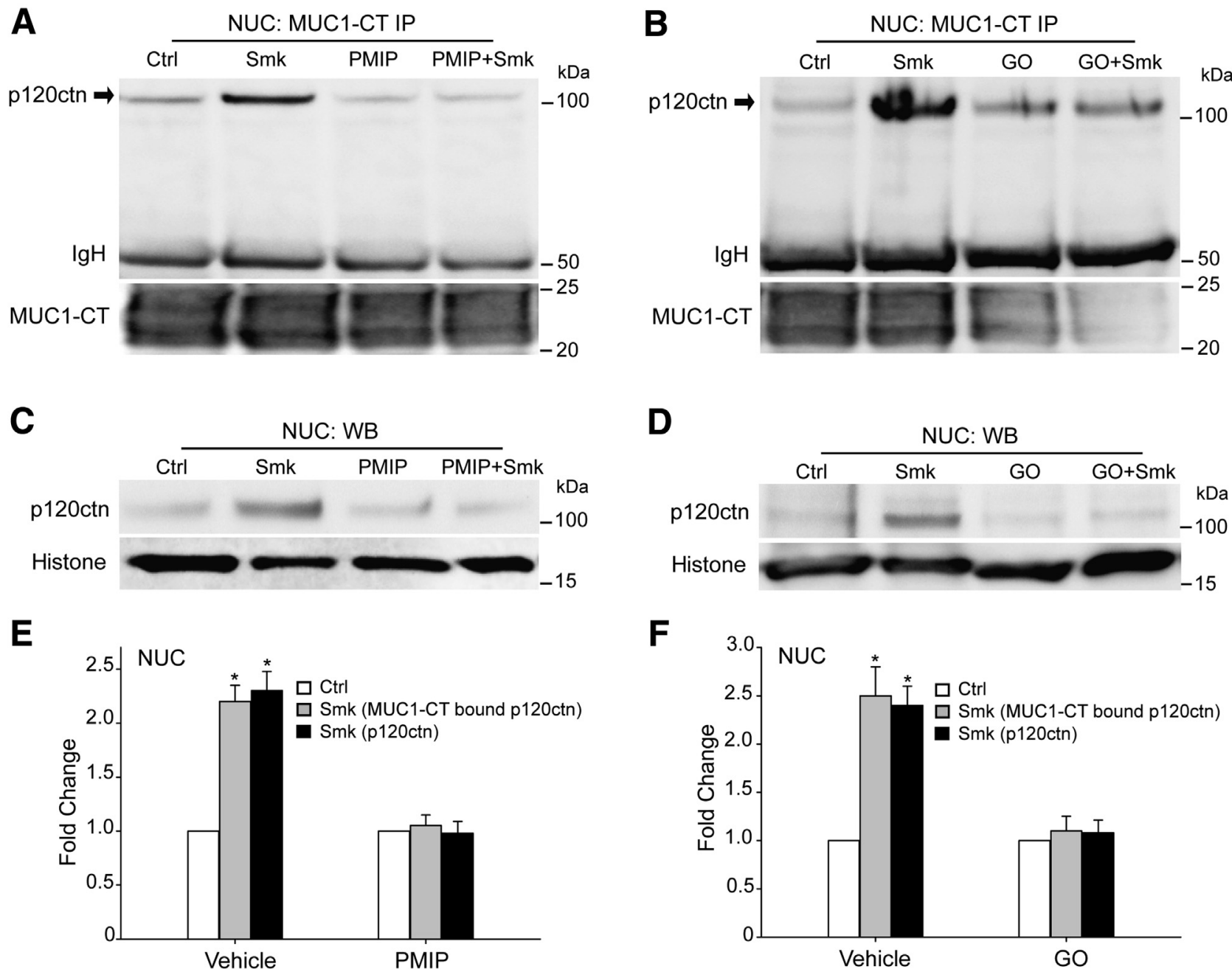

Figure 2 PMIP and G0 abolish smoke-induced nuclear p120ctn/MUC1-CT interaction and nuclear translocation of p120ctn in pseudostratified HBE cells. A-F: The cells were preincubated overnight with $50 \mu \mathrm{mol} / \mathrm{L}$ PMIP, $20 \mu \mathrm{mol} / \mathrm{L} \mathrm{G0}$, or vehicle control (water) before exposure to Ctrl or Smk in the presence of $50 \mu \mathrm{mol} / \mathrm{L}$ PMIP, $20 \mu \mathrm{mol} / \mathrm{L} \mathrm{G0}$, or water for 4 hours. NUC fractions were obtained. A and B: MUC1-CT IPs were probed for p120ctn and MUC1-CT. Equal loading was confirmed by IgH bands. $\mathbf{C}$ and $\mathbf{D}$ : NUC fractions were probed for p120ctn. Histone bands served as loading control. $\mathbf{E}$ and $\mathbf{F}$ : Densitometric quantitation of MUC1-CT-bound p120ctn and p120ctn in NUC of Smk- and/or PMIP-treated cells (A and C) and NUC of Smk- and/or G0-treated cells (B and D) was normalized to those in untreated Ctrl (designated as onefold). Data are expressed as means \pm SEM fold change. ${ }^{*} P<0.05$. Ctrl, smoke-free medium; G0, G0-201; HBE, human bronchial epithelial; IgH, immunoglobulin heavy chain; IP, immunoprecipitate; MUC1-CT, cytoplasmic tail of mucin-1; NUC, nuclear; p120ctn, p120-catenin; PMIP, MUC1 inhibitory peptide; Smk, smoke-concentrated medium; WB, Western blot analysis. 

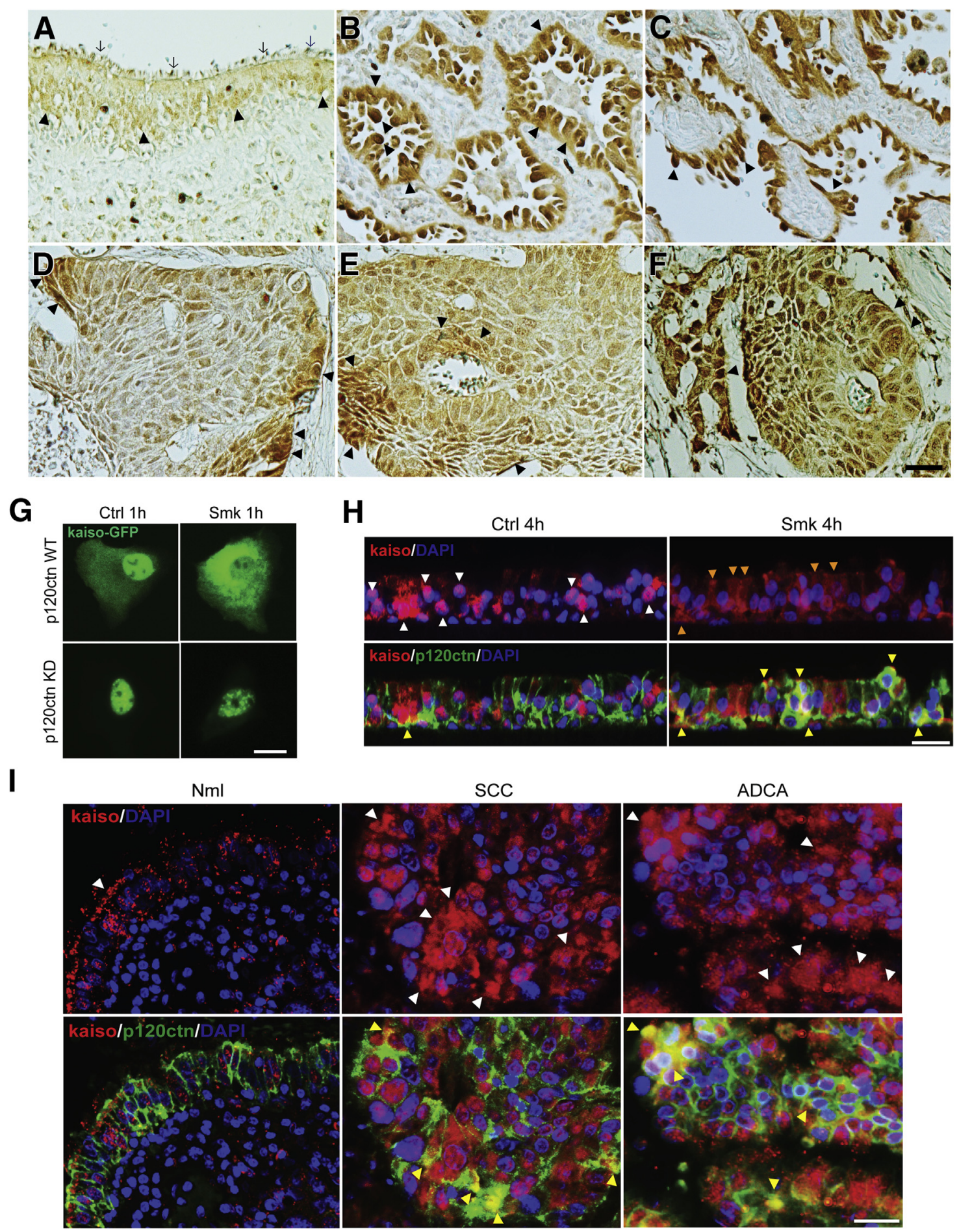

Figure 3 Smoke promotes nuclear-to-cytoplasmic trafficking of kaiso and kaiso/p120ctn colocalization. A-F: Increased cytoplasmic and nuclear expression of kaiso in lung carcinoma. Immunohistochemistry revealed heterogeneous expression of kaiso in the cytoplasm and nuclei in normal bronchial epithelium (A), lung adenocarcinoma (B and $\mathbf{C}$ ), and lung squamous cell carcinoma (D-F). Black arrows in $\mathbf{A}$ point to ciliated epithelial cells. Black arrowheads in $\mathbf{A}-\mathbf{F}$ point to strong kaiso staining in the nucleus and/or cytoplasm of cells. G: Kaiso-GFP plasmid (green) and scrambled siRNA were cotransfected into primary HBE cells by electroporation (p120ctn WT). Alternatively, kaiso-GFP vector (green) and p120ctn siRNA were cotransfected in HBE cells (p120ctn KD). At 48 hours after transfection, the cells were exposed to Ctrl or Smk medium for 1 hour before images were obtained. H: Immunostaining of kaiso (red) and p120ctn (green) in polarized HBE cells exposed to 4 hours of Ctrl or Smk. Cell nuclei were visualized with DAPI (blue). White arrowheads point to kaiso in the nuclei (purple signals generated by overlying of red/blue signals). Orange arrowheads point to kaiso in the cytoplasm. Yellow arrowheads point to colocalization of p120ctn and kaiso in the cytoplasm (yellow signals generated by overlying of green/red signals) and nuclei (white signals generated by overlying of green/red/blue signals). I: Immunostaining of kaiso (red) and p120ctn (green) in $\mathrm{Nml}$ bronchial epithelium, lung SCC and ADCA. White arrowheads indicate kaiso expression in the cytoplasm, yellow arrowheads indicate colocalization of kaiso and p120ctn (yellow signals generated by overlying of red/green signals). Scale bars $=50 \mu \mathrm{m}(\mathbf{A}-\mathbf{F}, \mathbf{H}$, and I); $20 \mu \mathrm{m}(\mathbf{G})$. ADCA, adenocarcinoma; Ctrl, smoke-free medium; GFP, green fluorescent protein; HBE, human bronchial epithelial; KD, knockdown; Nml, normal; p120ctn, p120-catenin; SCC, squamous cell carcinoma; Smk, smoke-concentrated medium; WT, wild type. 
MUC1-CT forms a barrier along the apical cell membrane of the normal bronchial epithelium, whereas AJ proteins p120ctn, $\beta$-ctn and E-cad are localized to subapical intercellular junctions (Figure 1E). In contrast, human specimens of SCC and ADCA, two forms of non-small cell lung cancer strongly linked with smoking, demonstrate colocalization of MUC1-CT and p120ctn in the cytoplasm and the apical/basolateral membranes (Figure 1E), as well as reduced or absent $\mathrm{p} 120 \mathrm{ctn} / \beta \mathrm{ctn} / \mathrm{E}-\mathrm{cad}$ from intercellular AJs (Figure 1E).

\section{Smoke-Induced NUC Translocation of p120ctn Depends} on MUC1-CT/p120ctn Interaction and NUC Localization of MUC1-CT

Previously, we demonstrated a smoke-induced, time-dependent increase of MUC1-CT/p120ctn complexes that were localized to the cytoplasm and intercellular junctions of fully differentiated, polarized, primary $\mathrm{HBE}$ cells. ${ }^{4}$ Because overexpression of MUC1-CT has been reported to increase NUC accumulation of $120 \mathrm{ctn},{ }^{24}$ we hypothesized that MUC1-CT may serve as a chaperone that escorts p120ctn to the nucleus of polarized HBE cells in response to smoke.

We thereby examined whether smoke promoted complex formation between MUC1-CT and p120ctn in the nucleus of HBE cells. Consistent with MUC1-CT/p120ctn complex formation in the cytoplasm and junctions, ${ }^{4}$ immunoblotting of NUC fractions from HBE cell lysates revealed a 2.2- to 2.5 -fold increase of MUC1-CT-bound p120ctn after 4 hours of smoke exposure versus control cells (Figure 2, A and B). We next examined whether blocking MUC1-CT/ p120ctn complex formation would prevent smoke-induced NUC translocation of p120ctn. In previous studies, we showed that PMIP, a MUC1 inhibitory peptide that contains the MUC1-CT/catenin binding site (SAGNGGSSLS), successfully blocked p120ctn/MUC1-CT interaction in total HBE cell lysates. ${ }^{4}$ Here, we showed that PMIP treatment also reduced MUC1-CT/p120ctn complex formation (Figure 2, A and E) and overall levels of p120ctn (Figure 2, $\mathrm{C}$ and $\mathrm{E}$ ) in the nucleus of HBE cells. These data suggest that smoke-promoted NUC translocation of p120ctn depends on MUC1-CT/p120ctn interaction.

The mechanism(s) by which MUC1-CT/p120ctn complex formation facilitates NUC trafficking of p120ctn after smoke exposure is unclear. In addition to aiding the release of p120ctn from $\mathrm{p} 120 \mathrm{ctn} / \beta$-ctn/E-cad complexes at $\mathrm{AJs},{ }^{4}$ MUC1-CT may directly speed NUC import of p120ctn. To test the latter hypothesis, we suppressed the NUC localization of MUC1-CT using another MUC1-CT inhibitory peptide GO. GO contains nine arginine residues (-[R $\left.]_{9^{-}}\right)$linked to the CQCRRKN motif of MUC1-CT required for its NUC import and thereby inhibits NUC entry of MUC1-CT. ${ }^{25}$ The $[\mathrm{R}]_{9}$ at

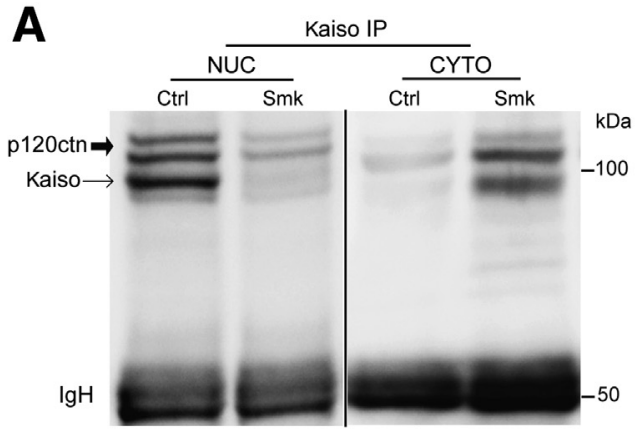

D

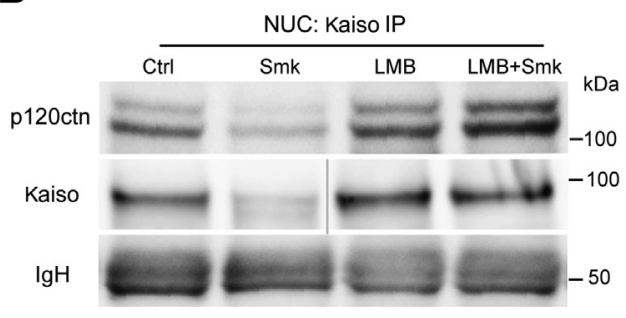

B

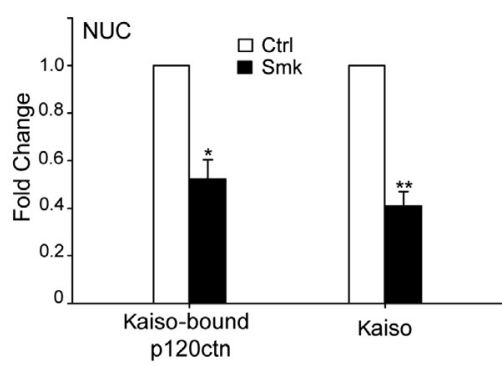

E

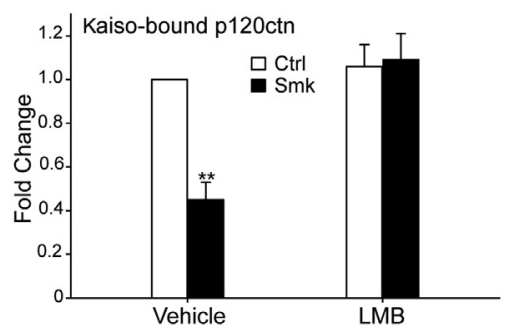

C

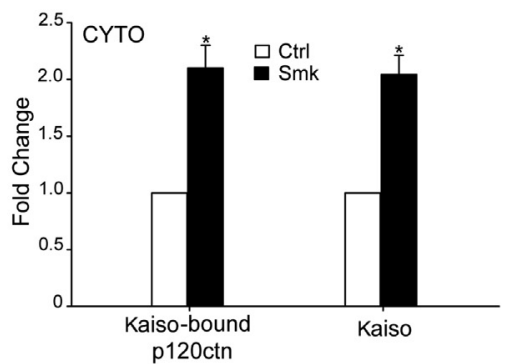

$\mathbf{F}$

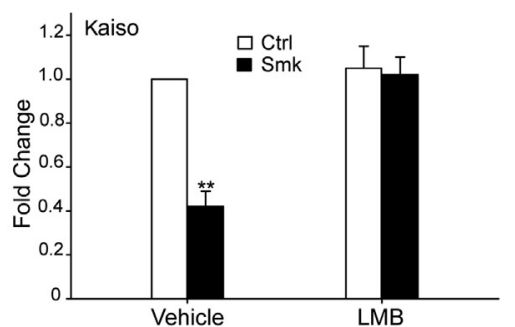

Figure 4 LMB abolishes smoke-induced NUC-to-CYTO shuttling of kaiso through mediating nuclear sequestration of p120ctn. A-C: Polarized HBE cells exposed to Ctrl or Smk for 4 hours. Kaiso IPs of NUC and CYTO fractions were probed with p120ctn and kaiso antibodies. IgH bands served as loading controls. Densitometric analysis of kaiso-bound p120ctn and kaiso in NUC (B) and CYTO (C) after smoke exposure was normalized to those in untreated Ctrl (designated as onefold). D-F: Polarized HBE cells were preincubated with $100 \mathrm{nmol} / \mathrm{L}$ LMB or vehicle control (equal volume of methanol) overnight before exposure to Ctrl or Smk in the presence of $100 \mathrm{nmol} / \mathrm{L} \mathrm{LMB}$ or methanol for 4 hours. Kaiso IPs of NUC fractions were probed with p120ctn and kaiso. The kaiso bands have been reorganized from the same gel. IgH bands were used as loading controls. Densitometric quantitation of kaiso-bound p120ctn $(\mathbf{E})$ and kaiso levels $(\mathbf{F})$ of Smktreated cells was normalized to those in Ctrl cells (designated as onefold). Data are expressed as means \pm SEM fold changes. ${ }^{*} P<0.05,{ }^{* *} P<0.01$. Ctrl, smoke-free medium; CYTO, cytoplasmic; HBE, human bronchial epithelial; IgH, immunoglobulin heavy chain; IP, immunoprecipitate; LMB, leptomycin-B; NUC, nuclear; p120ctn, p120-catenin; Smk, smoke-concentrated medium. 
the $\mathrm{N}$-terminus has been shown to confer cell penetration. ${ }^{26}$ Polarized HBE cells were preincubated with $20 \mu \mathrm{mol} / \mathrm{L}$ GO or vehicle control overnight before treatment with control medium (Ctrl) or Smk for 4 hours. Consistent with GO's established role in mediating NUC entry of MUC1-CT, NUC levels of MUC1-CT were reduced in GO-treated cells (Figure 2B). Correspondingly, GO abolished smokepromoted MUC1-CT/p120ctn complex formation in the nucleus (Figure 2, B and F) and down-regulated NUC p120ctn levels after smoke exposure (Figure 2, D and F) while having no effect on smoke-induced MUC1-CT/p120ctn interaction in the cytoplasm (data not shown). These data confirmed MUC1-CT's role as a chaperone facilitating smoke-induced junctional-to-NUC trafficking of p120ctn. This role as NUC chaperone in response to smoke is similar to that of fellow AJ protein $\beta$-ctn as we previously described. ${ }^{3}$
A
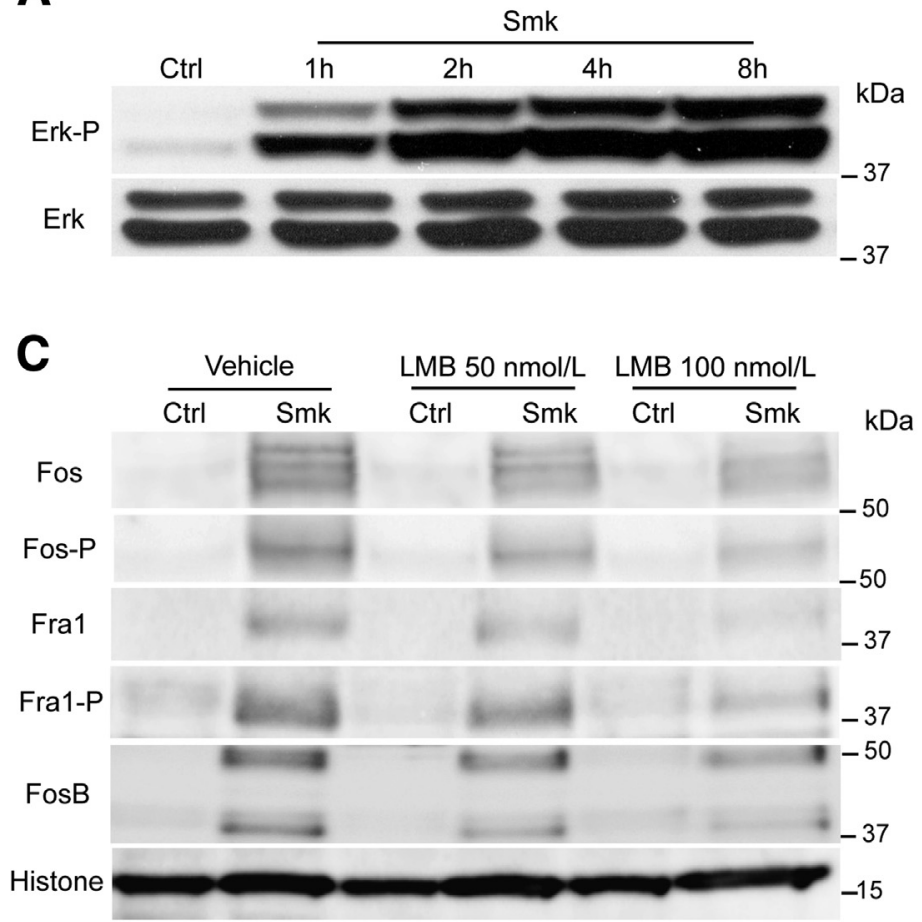

$\mathbf{E}$

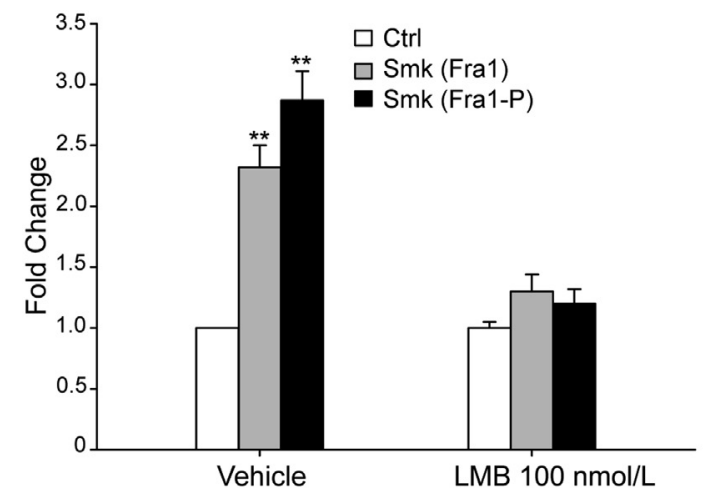

B

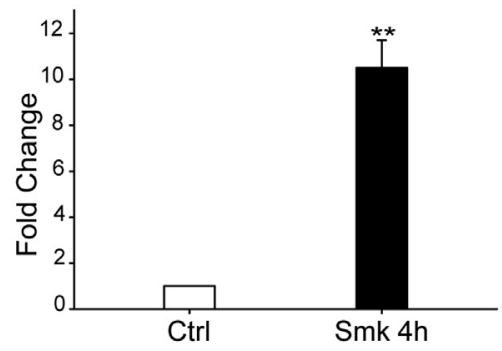

D

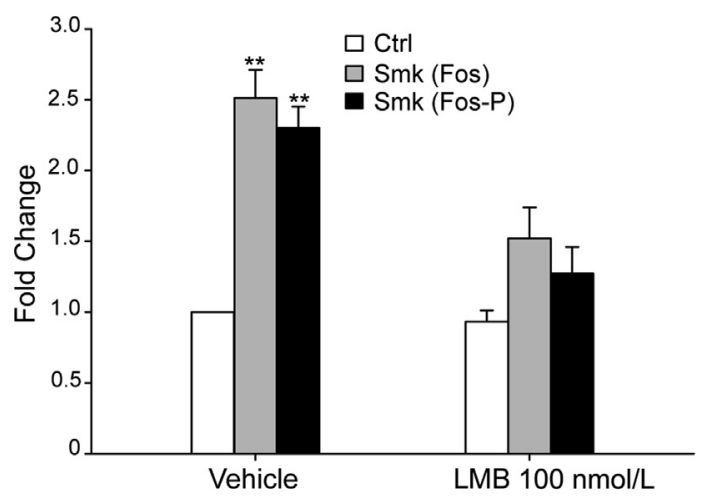

$\mathbf{F}$

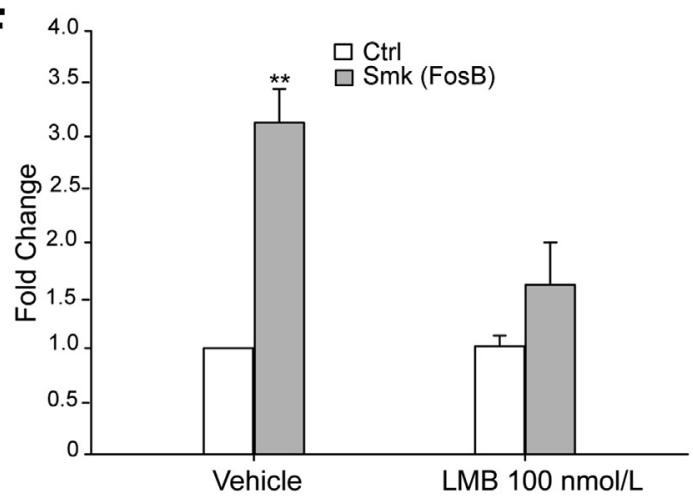

Figure 5 LMB abolished smoke-induced expression and activation of Fos, Fra1, and FosB downstream of Erk signaling in pseudostratified HBE cells. A: Smoke promotes time-dependent phosphorylation (activation) of Erk. HBE cells exposed to Smk were harvested at indicated time points. Cell lysates were analyzed by Western blot analyses probed with Erk-P antibody. Erk bands served as loading control. B: Quantitation of Erk-P after 4 hours of Smk exposure was normalized to untreated Ctrl (designated as onefold). C-F: Smoke induces dose-dependent up-regulation and activation of Fos, Fra1, and FosB in polarized HBE cells. The cells were treated with Ctrl or Smk for 4 hours in the presence of LMB or vehicle control as described above. C: Nuclear fractions of cell lysates were obtained and analyzed by Western blot analyses probed with Fos, Fos-P, Fra1, Fra1-P, and FosB antibodies. Histone bands served as loading control. D-F: Densitometric quantitation of Fos and Fos-P (D), Fra1 and Fra1-P (E), and FosB (F) in Smk- and/or LMB-treated cells was normalized to those in untreated Ctrl (designated as onefold). Data are expressed as means \pm SEM fold change. ${ }^{* *} P<0.01$. Ctrl, smoke-free medium; Erk, P44/42 mitogen-activated kinase; Erk-P, $\mathrm{Thr}^{202} / \mathrm{Tyr}^{204}$-phosphorylated $\mathrm{p} 44 / 42$ mitogen-activated kinase; Fos- $\mathrm{P}, \mathrm{Ser}^{32}$-phosphorylated Fos; Fra1-P, Ser ${ }^{265}$-phosphorylated Fra1; HBE, human bronchial epithelial; LMB, leptomycin-B; Smk, smoke-concentrated medium. 
A

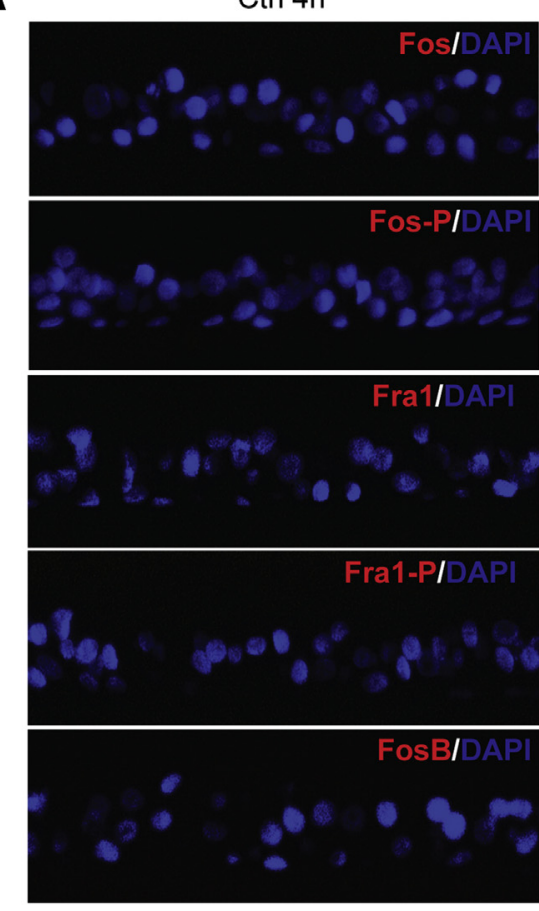

Smk 4h

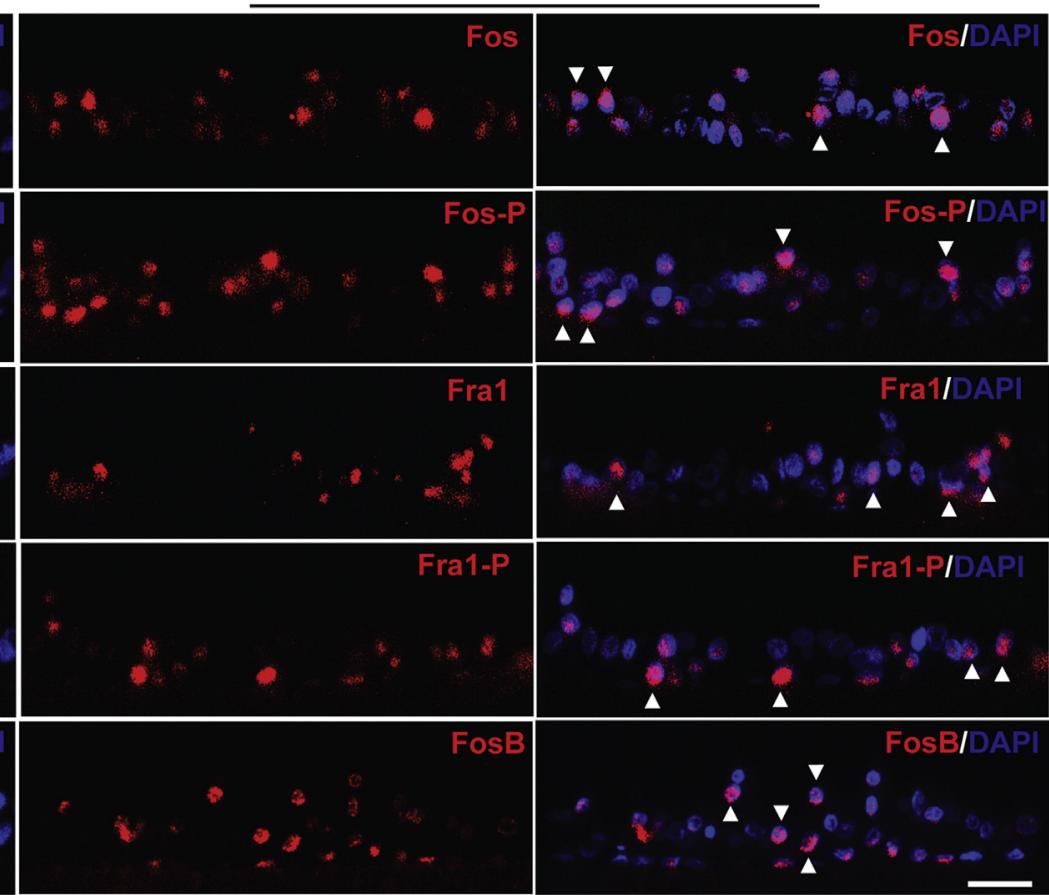

B

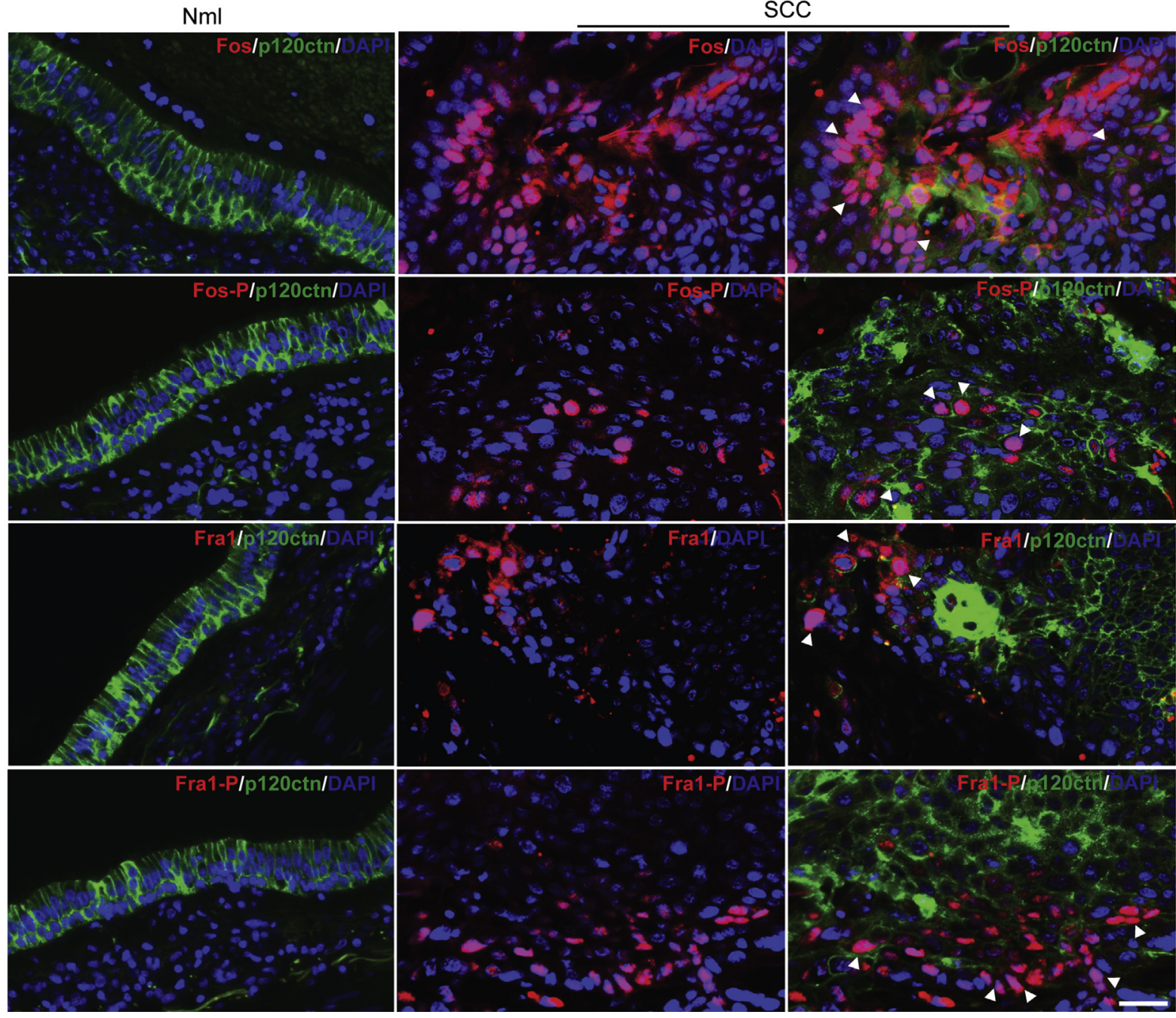


Smoke Promotes Kaiso/p120ctn Complex Formation and Mediates NUC-to- CYTO Trafficking of Kaiso

After smoke-provoked AJ disruption, junctional p120ctn was aberrantly translocated to the cytoplasm and nucleus of HBE cells (Figures 1 and 2). It is unclear what role p120ctn plays in lung carcinogenesis after it is driven into the nucleus by smoke-induced MUC1-CT/p120ctn interaction. Interestingly, we noted the presence of kaiso, an identified transcription inhibitor that binds to p120ctn, ${ }^{10}$ and displayed a similar CYTO /NUC localization pattern as p120ctn in human lung carcinoma specimens (Figure 1E). Kaiso was weakly expressed in the cytoplasm and nucleus of normal bronchial epithelium (Figure 3A). Kaiso expression was robustly and diffusely elevated in the cytoplasm while moderately increased in the nucleus of lung ADCA (Figure 3, B and C). Similarly, kaiso levels were increased in the cytoplasm and nucleus of lung SCC and were dramatically elevated at the leading edge of bulky mass (Figure 3D), perivascular areas (Figure 3E), and metastatic foci in mesenchyme (Figure 3F). We hypothesized that p120ctn may play a role in lung carcinogenesis through mediating the NUC-to-CYTO translocation of kaiso, where CYTO kaiso is unable to bind DNA and thereby cannot function as a transcription inhibitor.

To investigate whether smoke mediated the rapid NUC exit of kaiso, we transfected primary HBE cells with a kaiso-GFP construct. Kaiso-GFP was localized in both cytoplasm and NUC compartments in untreated wild-type cells, whereas it completely diffused out of the nucleus and aggregated in the cytoplasm after 1-hour of smoke exposure (Figure 3G). p120ctn was knocked down (p120ctn knockdown) in HBE cells by cotransfected p120ctn siRNAs as we previously described. ${ }^{2}$ In p120ctn knockdown cells, kaiso was exclusively localized in the nucleus of control cells and failed to enter the cytoplasm after 1 hour of smoke treatment (Figure 3G). Consistently, endogenous kaiso in pseudostratified HBE cells behaved similarly to overexpressed kaiso-GFP in response to smoke. As shown in Figure 3H, kaiso was mostly localized in the nucleus, whereas it was rapidly translocated into the cytoplasm after 4 hours of smoke exposure. Moreover, immunostaining revealed colocalization of kaiso and p120ctn in the cytoplasm and nucleus of polarized HBE cells in response to Smk (Figure 3H). Similarly, immunostaining revealed colocalization of kaiso and p120ctn in the cytoplasm and nucleus of lung SCC and ADCA specimens (Figure 3I). These data led us to investigate the potential interaction between p120ctn and kaiso in response to smoke.

\section{Smoke-Induced NUC-to-CYTO Shuttling of Kaiso Depends on p120ctn/Kaiso Complex Formation}

Immunoprecipitates of kaiso were obtained from NUC and CYTO fractions of polarized HBE cells exposed to Ctrl or Smk medium for 4 hours. WB analysis revealed a rapid NUCto-CYTO trafficking of kaiso/p120ctn complexes in response to smoke (Figure 4A). In the nucleus, there was a $58 \%$ decrease of kaiso corresponding to a 55\% reduction of kaisobound p120ctn (Figure 4B). In the cytoplasm, there was a twofold elevation of kaiso compared with a 2.1-fold increase of kaiso-bound p120ctn (Figure 4C). These data suggest a significant association between kaiso levels and kaiso-bound p120ctn in both CYTO and NUC compartments.

Next, we investigated whether smoke-induced NUC exit of kaiso was suppressed by retaining p120ctn in the nucleus using LMB. LMB inhibits chromosomal region maintenance receptor-mediated NUC export and results in NUC sequestration of proteins with NUC export signals. ${ }^{27}$ With the understanding that p120ctn has an NUC export signal but kaiso does not, LMB would sequester kaiso in the nucleus during smoke exposure if NUC exit of kaiso depends on kaiso/p120ctn interaction. Immunoprecipitates with kaiso were obtained from NUC fractions of polarized HBE cells exposed to Ctrl or Smk for 4 hours after pretreatment with $100 \mathrm{nmol} / \mathrm{L}$ LMB overnight. LMB retained p120ctn in the nucleus and thereby completely abolished NUC-to-CYTO translocation of kaiso in response to smoke (Figure 4D). Densitometric analysis revealed a complete sequestration of kaiso and kaiso-bound p120ctn in the nucleus of LMB-treated cells in comparison with a significant reduction of kaiso/p120ctn complexes in the nucleus of control cells (Figure 4, E and F). These data suggested that smoke-promoted NUC-to-CYTO translocation of kaiso likely depends on formation of kaiso/ p120ctn complexes.

\section{Smoke-Induced Expression of Transcription Factors Fos, Fra1, and FosB Depends on NUC-to-CYTO Translocation of Kaiso}

We next investigated the potential consequence of trapping kaiso in the CYTO pool of p120ctn/kaiso complexes after smoke exposure. We hypothesized that smoke

\footnotetext{
Figure 6 Increased expression and activation of Fos, Fra1, and FosB in smoke-treated HBE cells and lung SCC. A: Polarized HBE cells were treated with Ctrl or Smk for 4 hours. Immunostaining of HBE cells with antibodies against Fos (red, row 1), Fos-P (red, row 2), Fra1 (red, row 3), Fra1-P (red, row 4), and FosB (red, row 5). Cell nuclei were counterstained with DAPI (blue). White arrowheads point to nuclear localization of the above proteins (purple signals generated by overlying red and blue signals). B: Immunostaining of lung SCC with antibodies against Fos (red, top row), Fos-P (red, second row), Fra1 (red, third row), Fra1-P (red, bottom row), and p120ctn (green, all panels). Cell nuclei were counterstained with DAPI (blue). White arrowheads point to nuclear localization of Fos/Fos-P/Fra1/Fra1-P (purple signals generated by overlying red and blue signals) at the perivascular area or leading edge of tumor foci. Scale bars $=50$ $\mu \mathrm{m}$. Ctrl, smoke-free medium; HBE, human bronchial epithelial; Fos-P, Ser ${ }^{32}$-phosphorylated Fos; Fra1-P, Ser ${ }^{265}$-phosphorylated Fra1; Nml, normal; p120ctn, p120-catenin; SCC, squamous cell carcinoma; Smk, smoke-concentrated medium.
} 
provoked the expression of a subset of preoncogenic genes that were normally suppressed by NUC kaiso through mediating NUC-to-CYTO translocation of kaiso/p120ctn complexes.

Smoke stimulated a time-dependent increase of Erk-P, occurring as early as 1 hour, achieving a 10.5 -fold increase by 4 hours and persisting through 8 hours (Figure 5, A and B). Because sustained Erk activation was a prerequisite for expression of certain activator protein 1 transcription factors, ${ }^{28}$ we examined whether smoke up-regulated transcription of several members of the c-Fos family (typical of activator protein 1 genes that are expressed rapidly). In conjunction with the time-dependent increase of Erk-P, smoke induced a time-dependent increase of Fos, Fra1, and FosB mRNAs (Supplemental Figure S1A). Because kaiso has been reported to directly repress transcription of cFos, ${ }^{13}$ we next examined whether LMB treatment would suppress smoke-induced transcription of c-Fos genes by retaining p120ctn/kaiso complexes in the nucleus. As expected, LMB successfully inhibited the up-regulation of Fos, Fra1, and FosB mRNAs in response to smoke (Supplemental Figure S1B).

Consistent with increased Fos, Fra1, and FosB mRNAs, WB analysis of the NUC fractions revealed dose-dependent up-regulation of Fos, Fos-P, Fra1, Fra1-P, and FosB proteins in the nucleus of polarized HBE cells after 4 hours of smoke exposure (Figure 5C). Densitometry analysis revealed that smoke provoked a 2.5 -fold increase of Fos in conjunction with a 2.3-fold increase of Fos-P (Figure 5D), a 2.3 -fold increase of Fra1 in conjunction with a 2.8-fold increase of Fra1-P (Figure 5E), and a 3.1-fold increase of FosB (Figure 5F). These data suggested that NUC Fos and Fra1 were predominantly in their activated (ie, phosphorylated) state in the nucleus of smoke-treated cells. Moreover, consistent with the results of transcript studies (Supplemental Figure S1B), LMB treatment completely abolished smoke-induced expression of Fos, Fos-P, Fra1, Fra1-P, and FosB protein by sequestrating p120ctn/kaiso complexes in the nucleus (Figure 5, C-F), suggesting Fos, Fra1, and FosB are likely kaiso's target genes in response to smoke exposure.

Finally, immunostaining confirmed up-regulated Fos, Fos-P, Fra1, Fra1-P, and FosB in polarized HBE cells after 4 hours of smoke exposure (Figure 6A). In consistent, immunostaining revealed increased expression of Fos, Fos-P, Fra1, and Fra1-P in lung SCC in comparison with adjacent normal bronchial epithelium (Figure 6B). In line with the distribution pattern of CYTO kaiso in lung SCC (Figure 3, D-F), the expression of c-Fos proteins was extremely robust at perivascular areas, metastatic foci, and leading edge of carcinoma mass (Figure 6B). These data support the possibility of down-regulating preoncogenic genes (ie, c-Fos transcription factors) through suppressing NUC exit of kaiso, thereby preventing lung carcinogenesis in smoker's bronchial epithelium.

\section{Discussion}

According to our data, three sequential molecular events induced by smoke provoked the CYTO accumulation of transcriptional repressor, kaiso, to facilitate the deinhibition of kaiso's protumor target genes (Figure 7). First, MUC1$\mathrm{CT}$ acts as a chaperone facilitating smoke-induced NUC entry of p120ctn. Second, smoke induces kaiso's release from its DNA binding sites in the nucleus and promotes its CYTO translocation through an interaction with NUC p120ctn. Finally, smoke induces transcription of protumor genes that are normally suppressed by NUC kaiso.

We revealed that smoke-induced NUC entry of p120ctn depends on MUC1-CT/p120ctn interaction. We previously reported that smoke-induced MUC1-CT/p120ctn interaction competed with AJ E-cad for p120ctn binding, which exposed the ubiquitination sites of E-cad and led to its degradation during smoke exposure. ${ }^{4}$ Here, we confirmed MUC1-CT/ p120ctn colocalization and loss of p120ctn/ $\beta$-ctn/E-cad from AJs in human lung SCC and ADCA specimens (Figure 1E). Consistent with this finding, MUC1-CT/p120ctn interaction has been reported to modulate distinct features of cell adhesion, motility, and metastasis of pancreatic cancer cells. ${ }^{29}$ Adding to this work, we showed that smoke-induced MUC1-CT/p120ctn interaction was essential for regulating the NUC entry of p120ctn (Figures 1 and 2). This finding is in line with our previous studies on $\beta$-ctn, where its interaction with MUC1-CT facilitated $\beta$-ctn's import into the nucleus. ${ }^{3}$ Here, smoke promoted a time-dependent NUC accumulation of MUC1-CT and p120ctn (Figure 1, A and B), with extremely rapid CYTO-to-NUC translocation of MUC1CT-RFP seen in 5 minutes of smoke exposure (Figure 1, C and D). The essential nature of MUC1-CT's role in this process was demonstrated using MUC1-CT decoy peptide PMIP, which blocked smoke-provoked MUC1-CT/p120ctn complex formation in total lysates ${ }^{4}$ and nucleus (Figure 2A), as well as significantly reducing NUC p120ctn levels in the nucleus (Figure 2, C and E). In concert with our data, overexpression of MUC1-CT increased NUC p120ctn accumulation through MUC1-CT/p120ctn interaction in breast cancer cells. ${ }^{24}$ Moreover, rapid NUC translocation of yellow fluorescent protein-tagged MUC1-CT from cytoplasm and cell surface has also been reported in pancreatic cancer cells within minutes of hepatocyte growth factor stimulation. ${ }^{30}$

The mechanism whereby MUC1-CT assisted in the NUC localization of MUC1-CT/p120ctn complexes after smoke exposure was unclear. Previous work showed that MUC1-CT's trafficking to the nucleus was due to the presence of a nonclassic NUC localization signal (NLS), the RRK motif that interacts with importin- $\beta$ and nucleoporin p62. ${ }^{31}$ In contrast, p120ctn contains two classic $\mathrm{NLSs}^{32}$ and has been shown to be imported into the nucleus by first binding to importin- $\alpha$ before its association with importin- $\beta$. $^{33}$ MUC1-CT also contains a CQC motif mediating its oligomerization, which is essential to MUC1-CT's association with nucleoporin $\mathrm{p}^{3}{ }^{31}$ These 


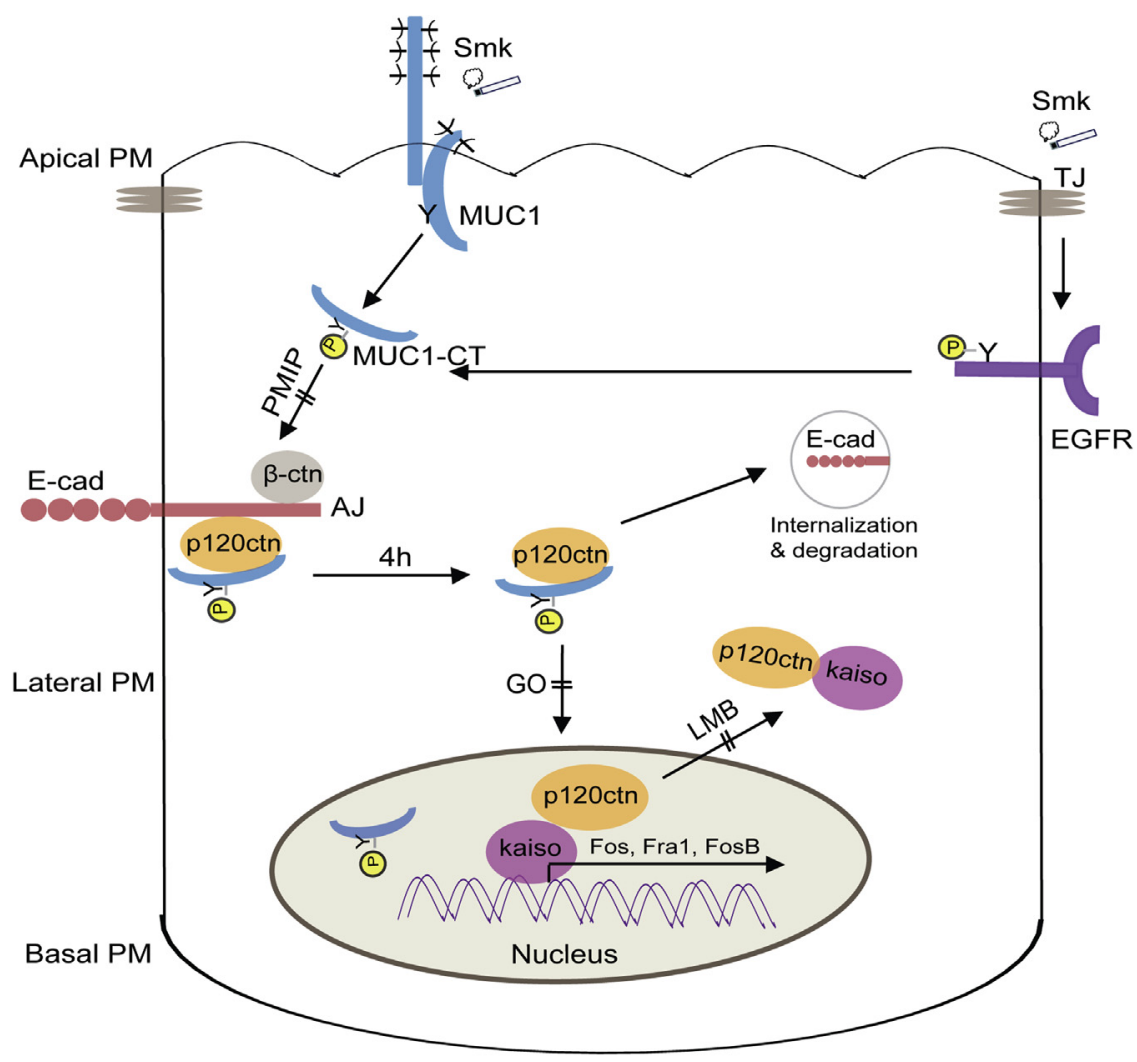

Figure 7 Schematic representation of the molecular events that initiate carcinogenesis in airway epithelial cells exposed to cigarette smoke. In polarized human airway epithelial cells, MUC1 is localized on the apical PM where it is spatially segregated from AJ proteins on the basolateral PM. Cigarette Smk activates epidermal growth factor receptor on the basolateral $P M$ to initiate the $Y$ - $P$ of MUC1-CT. ${ }^{4}$ Activation of MUC1-CT through Y-P promotes its interaction with $\mathrm{AJ}$ protein, p120ctn. Through this interaction, MUC1-CT competes with E-cad for p120ctn binding and disrupts the integrity of the $\mathrm{AJ}$ complex (p120ctn/E-cad/ $\beta$ ctn). ${ }^{4} \mathrm{E}$-cad is lost from the PM, internalized, and degraded, marking the initiation of EMT. MUC1-CT/ p120ctn complex formation in response to Smk also appears to mediate the nuclear import of p120ctn where it interacts with the transcriptional repressor, kaiso. Kaiso's interaction with p120ctn coincides with its accumulation in the cytoplasm and increased expression of protumor target genes, such as Fos, FosB, and Fra1, which are normally inhibited by kaiso. AJ, adherens junction; E-cad, epithelial cadherin; EGFR, epidermal growth factor receptor; EMT, epithelial-mesenchymal transition; G0, G0-201; MUC1, mucin-1; MUC1-CT, cytoplasmic tail of MUC1; PM, plasma membrane; p120ctn, p120-catenin; Smk, smoke; TJ, tight junction; $\mathrm{Y}-\mathrm{P}$, tyrosine phosphorylation; $\beta$-ctn, $\beta$-catenin. features allow for MUC1-CT's NUC cotranslocation with several protein partners, including p120ctn (Figure 2, A and B), $\beta$-ctn, ${ }^{34}$ epidermal growth factor receptor, ${ }^{35}$ and estrogen receptor- $\alpha{ }^{36}$ GO contains a poly Arg transduction domain in tandem with the RRKCQCN motif of MUC1-CT. ${ }^{25}$ In contrast to PMIP, which binds p120ctn, GO binds to MUC1-CT's CQC motif and thereby prevents its oligomerization and subsequent NUC translocation. We showed that GO down-regulated the NUC levels of MUC1-CT and MUC1-CT/p120ctn complexes (Figure 2B), which reduced NUC p120ctn during smoke exposure (Figure 2, D and F). Moreover, NUC localization of p120ctn was unaffected by simultaneous deletion of both NLS domains, whereas it was abolished by deletion of armadillo repeat 3 or 5 despite of the presence of intact NLSs. ${ }^{37}$ These findings are consistent with previous reports of heregulininduced (an epidermal growth factor receptor ligand) MUC1$\mathrm{CT} / \gamma$-catenin $(\gamma$-ctn) interaction and targeting of the complex to the nucleolus, which was abrogated by mutation of MUC1CT's RRK motif. ${ }^{38}$ In addition, p120ctn's armadillo domains 1,3 to 5 , and 8 have recently been reported to mediate $\mathrm{p} 120 \mathrm{ctn} /$ MUC1-CT interaction in pancreatic cancer cells. ${ }^{29}$ Thus, we propose that MUC1-CT/p120ctn interaction facilitates NUC p120ctn accumulation through three underlying mechanisms. First, MUC1-CT mediates p120ctn's release from AJ E-cad/ catenin complexes during smoke exposure. ${ }^{4}$ Second, MUC1CT increases p120ctn's stability in response to smoke, thereby preventing its degradation during NUC entry. ${ }^{39}$ Third, MUC1-CT/p120ctn interaction directs NUC localization of p120ctn through MUC1-CT/importin- $\beta$ association. These smoke-mediated events are supported by our data and that of others showing E-cad overexpression abolished p120ctn's NUC entry whereas E-cad deletion increased its NUC accumulation in breast cancer cells. ${ }^{40}$

Subsequent to p120ctn's NUC translocation in response to smoke, we revealed its interaction with transcriptional repressor, kaiso. The interaction between p120ctn and kaiso assisted in shuttling kaiso out of the nucleus and trapping it in the cytoplasm. In support of this finding, we examined human specimens of lung SCC and ADCA, which revealed that kaiso's CYTO or NUC distribution (Figure 3, A-F) closely mimicked the aberrant CYTO or NUC localization of p120ctn (Figures $1 \mathrm{E}$ and $3 \mathrm{I}$ ). We recapitulated in $\mathrm{HBE}$ cells where smoke-induced NUC-to-CYTO shuttling of kaiso in complex with p120ctn occurred within hours of exposure (Figure 3, G and $\mathrm{H}$, and Figure 4, A-C). These data suggest that smoke-induced p120ctn/kaiso interaction facilitates lung carcinogenesis through the after mechanisms. First, smoke promotes NUC entry of p120ctn (Figure 1, A and B). Second, smoke-induced NUC p120ctn/ kaiso interaction (Figure 4, A and B) facilitates kaiso's dissociation from its DNA targets. The binding site of p120ctn has been mapped into kaiso's zinc finger domain, which is important for associating kaiso with DNA.' Moreover, the presence of steric competition between p120ctn and DNA for kaiso binding has been revealed by in vivo chromatin immunoprecipitation and in vitro electrophoretic mobility shift assay. ${ }^{14}$ Third, p120ctn assists in shuttling kaiso out of the nucleus in response to smoke. 
Although kaiso has a NLS, its NUC export signal remains undiscovered. ${ }^{41}$ Although most kaiso is localized in the nucleus of polarized HBE cells (Figure $3 \mathrm{H}$ ) and normal bronchi (Figure 3A), LMB suppressing chromosomal region maintenance-mediated NUC export of p120ctn retained kaiso in the nucleus (Figure 4, D-F). Finally, p120ctn retains kaiso in the CYTO reservoir (Figure 4, A and C) for degradation or future reentering of nucleus, thereby abolishing kaiso's functional role as a transcriptional repressor. CYTO kaiso localization was reported to coincide with CYTO p120ctn in advanced-stage lung cancer and lymph node metastases, where it was associated with a significantly lower 5-year survival rate. ${ }^{42}$

Finally, we revealed a role for c-Fos members in the signaling pathway responsible for mediating the NUC exit of kaiso in response to smoke. Smoke up-regulated transcription (Supplemental Figure S1A) and expression (Figure 5, A and C) of Fos/Fos-P, Fra1/Fra1-P, and FosB downstream of Erk signaling in polarized HBE cells. Increased expression of Fos/Fos-P and Fra1/Fra1-P was also revealed in human lung SCC (Figure 6B). Mild increase of Fos transcription factors was detected in lung ADCA (data not shown), indicating kaiso may have activator protein 1 targets other than Fos members in these patients. Consistent with our results, smoke was previously reported to increase expression of Fos and Fra1 downstream of Erk signaling. ${ }^{43,44}$ Increased Fos was identified as a prognostic factor of lung cancer in addition to clinical staging. ${ }^{45}$ Furthermore, the smoke-induced increase of Fos/Fra1/FosB mRNAs (Supplemental Figure S1B) and proteins (Figure 5, C-F) was abolished using LMB to retain kaiso in the nucleus. Fos has previously been reported as kaiso's target gene, ${ }^{13}$ whereas Fra1 and FosB are likely repressed by kaiso. Whether members of the Fos family are direct targets of kaiso will be further investigated by chromatin immunoprecipitation assay.

Together with our previous work delineating the essential role of p120ctn's role in mediating the early stages of malignant transformation in response to cigarette smoke, ${ }^{1-5}$ this present study revealed the therapeutic potential of blocking p120ctn's interaction with kaiso as a novel approach to suppress the activation of protumor genes and premalignant changes in airways of smokers. Because secondhand smoking accounts for a high percentage of lung cancer in never smokers ${ }^{46}$ the study may also shed light in early treatment of lung cancer in never smokers with long-term exposure to environmental tobacco smoke.

\section{Acknowledgments}

We thank Dr. Sandra J. Gendler (Mayo Clinic College of Medicine, Arizona) for MUC1-CT 2 antibody and Dr. Brigitte E. Blackman (Center for Reproductive Sciences, University of California San Francisco) for helping with fluorescence microscopy.

\section{Supplemental Data}

Supplemental material for this article can be found at http://dx.doi.org/10.1016/j.ajpath.2016.08.011.

\section{References}

1. Zhang L, Gallup M, Zlock L, Finkbeiner W, McNamara NA: p120catenin modulates airway epithelial cell migration induced by cigarette smoke. Biochem Biophys Res Commun 2012, 417:49-55

2. Zhang L, Gallup M, Zlock L, Finkbeiner WE, McNamara NA: Rac1 and Cdc42 differentially modulate cigarette smoke-induced airway cell migration through p120-catenin-dependent and -independent pathways. Am J Pathol 2013, 182:1986-1995

3. Chen YT, Gallup M, Nikulina K, Lazarev S, Zlock L, Finkbeiner W, McNamara N: Cigarette smoke induces epidermal growth factor receptor-dependent redistribution of apical MUC1 and junctional betacatenin in polarized human airway epithelial cells. Am J Pathol 2010, $177: 1255-1264$

4. Zhang L, Gallup M, Zlock L, Basbaum C, Finkbeiner WE McNamara NA: Cigarette smoke disrupts the integrity of airway adherens junctions through the aberrant interaction of p120-catenin with the cytoplasmic tail of MUC1. J Pathol 2013, 229:74-86

5. Zhang L, Gallup M, Zlock L, Chen YT, Finkbeiner WE, McNamara NA: Pivotal role of MUC1 glycosylation by cigarette smoke in modulating disruption of airway adherens junctions in vitro. $\mathrm{J}$ Pathol 2014, 234:60-73

6. Kufe DW: Mucins in cancer: function, prognosis and therapy. Nat Rev Cancer 2009, 9:874-885

7. Miao Y, Liu N, Zhang Y, Liu Y, Yu JH, Dai SD, Xu HT, Wang EH p120ctn isoform 1 expression significantly correlates with abnormal expression of E-cadherin and poor survival of lung cancer patients. Med Oncol 2010, 27:880-886

8. Reynolds AB, Roczniak-Ferguson A: Emerging roles for p120-catenin in cell adhesion and cancer. Oncogene 2004, 23:7947-7956

9. Daniel JM, Reynolds AB: The catenin p120(ctn) interacts with Kaiso, a novel BTB/POZ domain zinc finger transcription factor. Mol Cell Biol 1999, 19:3614-3623

10. Daniel JM, Spring CM, Crawford HC, Reynolds AB, Baig A: The p120(ctn)-binding partner Kaiso is a bi-modal DNA-binding protein that recognizes both a sequence-specific consensus and methylated CpG dinucleotides. Nucleic Acids Res 2002, 30:2911-2919

11. Yoon HG, Chan DW, Reynolds AB, Qin J, Wong J: N-CoR mediates DNA methylation-dependent repression through a methyl $\mathrm{CpG}$ binding protein Kaiso. Mol Cell 2003, 12:723-734

12. Kim SW, Park JI, Spring CM, Sater AK, Ji H, Otchere AA, Daniel JM, McCrea PD: Non-canonical Wnt signals are modulated by the Kaiso transcriptional repressor and p120-catenin. Nat Cell Biol 2004, 6: 1212-1220

13. Park JI, Kim SW, Lyons JP, Ji H, Nguyen TT, Cho K, Barton MC, Deroo T, Vleminckx K, Moon RT, McCrea PD: Kaiso/p120-catenin and TCF/beta-catenin complexes coordinately regulate canonical Wnt gene targets. Dev Cell 2005, 8:843-854

14. van Roy FM, McCrea PD: A role for Kaiso-p120ctn complexes in cancer? Nat Rev Cancer 2005, 5:956-964

15. Liu Y, Dong QZ, Wang S, Xu HT, Miao Y, Wang L, Wang EH: Kaiso interacts with $\mathrm{p} 120$-catenin to regulate beta-catenin expression at the transcriptional level. PLoS One 2014, 9:e87537

16. Bitler BG, Menzl I, Huerta CL, Sands B, Knowlton W, Chang A Schroeder JA: Intracellular MUC1 peptides inhibit cancer progression. Clin Cancer Res 2009, 15:100-109

17. Liu X, Yuan Z, Chung M: MUC1 intra-cellular trafficking is clathrin, dynamin, and rab5 dependent. Biochem Biophys Res Commun 2008 376:688-693 
18. Filion GJ, Zhenilo S, Salozhin S, Yamada D, Prokhortchouk E, Defossez PA: A family of human zinc finger proteins that bind methylated DNA and repress transcription. Mol Cell Biol 2006, 26: 169-181

19. Schroeder JA, Thompson MC, Gardner MM, Gendler SJ: Transgenic MUC1 interacts with epidermal growth factor receptor and correlates with mitogen-activated protein kinase activation in the mouse mammary gland. J Biol Chem 2001, 276:13057-13064

20. Faigenbaum R, Haklai R, Ben-Baruch G, Kloog Y: Growth of poorly differentiated endometrial carcinoma is inhibited by combined action of medroxyprogesterone acetate and the Ras inhibitor Salirasib. Oncotarget 2013, 4:316-328

21. Simpkins F, Hevia-Paez P, Sun J, Ullmer W, Gilbert CA, da Silva T, Pedram A, Levin ER, Reis IM, Rabinovich B, Azzam D, Xu XX, Ince TA, Yang JY, Verhaak RG, Lu Y, Mills GB, Slingerland JM: Src Inhibition with saracatinib reverses fulvestrant resistance in ERpositive ovarian cancer models in vitro and in vivo. Clin Cancer Res 2012, 18:5911-5923

22. Han R, Huang S, Bao Y, Liu X, Peng X, Chen Z, Wang Q, Wang J, Zhang Q, Wang T, Zheng D, Yang W: Upregulation of SOX4 antagonizes cellular senescence in esophageal squamous cell carcinoma. Oncol Lett 2016, 12:1367-1372

23. Dieterich LC, Klein S, Mathelier A, Sliwa-Primorac A, Ma Q, Hong YK, Shin JW, Hamada M, Lizio M, Itoh M, Kawaji H, Lassmann T, Daub CO, Arner E, Carninci P, Hayashizaki Y, Forrest AR, Wasserman WW, Detmar M: DeepCAGE transcriptomics reveal an important role of the transcription factor MAFB in the lymphatic endothelium. Cell Rep 2015, 13:1493-1504

24. Li Y, Kufe D: The Human DF3/MUC1 carcinoma-associated antigen signals nuclear localization of the catenin p120(ctn). Biochem Biophys Res Commun 2001, 281:440-443

25. Raina D, Kosugi M, Ahmad R, Panchamoorthy G, Rajabi H, Alam M, Shimamura T, Shapiro GI, Supko J, Kharbanda S, Kufe D: Dependence on the MUC1-C oncoprotein in non-small cell lung cancer cells. Mol Cancer Ther 2011, 10:806-816

26. Raina D, Ahmad R, Joshi MD, Yin L, Wu Z, Kawano T, Vasir B, Avigan D, Kharbanda S, Kufe D: Direct targeting of the mucin 1 oncoprotein blocks survival and tumorigenicity of human breast carcinoma cells. Cancer Res 2009, 69:5133-5141

27. Zhang PX, Wang Y, Liu Y, Jiang GY, Li QC, Wang EH: p120-catenin isoform 3 regulates subcellular localization of Kaiso and promotes invasion in lung cancer cells via a phosphorylation-dependent mechanism. Int J Oncol 2011, 38:1625-1635

28. Balmanno K, Cook SJ: Sustained MAP kinase activation is required for the expression of cyclin D1, p21Cip1 and a subset of AP-1 proteins in CCL39 cells. Oncogene 1999, 18:3085-3097

29. Liu X, Yi C, Wen Y, Radhakrishnan P, Tremayne JR, Dao T, Johnson KR, Hollingsworth MA: Interactions between MUC1 and p120 catenin regulate dynamic features of cell adhesion, motility, and metastasis. Cancer Res 2014, 74:1609-1620

30. Singh PK, Behrens ME, Eggers JP, Cerny RL, Bailey JM, Shanmugam K, Gendler SJ, Bennett EP, Hollingsworth MA: Phosphorylation of MUC1 by Met modulates interaction with p53 and MMP1 expression. J Biol Chem 2008, 283:26985-26995
31. Leng Y, Cao C, Ren J, Huang L, Chen D, Ito M, Kufe D: Nuclear import of the MUC1-C oncoprotein is mediated by nucleoporin Nup62. J Biol Chem 2007, 282:19321-19330

32. Kelly KF, Spring CM, Otchere AA, Daniel JM: NLS-dependent nuclear localization of p120ctn is necessary to relieve Kaiso-mediated transcriptional repression. J Cell Sci 2004, 117:2675-2686

33. Stewart M: Molecular mechanism of the nuclear protein import cycle. Nat Rev Mol Cell Biol 2007, 8:195-208

34. Wen Y, Caffrey TC, Wheelock MJ, Johnson KR, Hollingsworth MA: Nuclear association of the cytoplasmic tail of MUC1 and beta-catenin. J Biol Chem 2003, 278:38029-38039

35. Bitler BG, Goverdhan A, Schroeder JA: MUC1 regulates nuclear localization and function of the epidermal growth factor receptor. J Cell Sci 2010, 123:1716-1723

36. Wei X, Xu H, Kufe D: MUC1 oncoprotein stabilizes and activates estrogen receptor alpha. Mol Cell 2006, 21:295-305

37. Roczniak-Ferguson A, Reynolds AB: Regulation of p120-catenin nucleocytoplasmic shuttling activity. J Cell Sci 2003, 116: 4201-4212

38. Li Y, Yu WH, Ren J, Chen W, Huang L, Kharbanda S, Loda M, Kufe D: Heregulin targets gamma-catenin to the nucleolus by a mechanism dependent on the DF3/MUC1 oncoprotein. Mol Cancer Res 2003, 1:765-775

39. Huang L, Chen D, Liu D, Yin L, Kharbanda S, Kufe D: MUC1 oncoprotein blocks glycogen synthase kinase 3beta-mediated phosphorylation and degradation of beta-catenin. Cancer Res 2005, 65: 10413-10422

40. van Hengel J, Vanhoenacker P, Staes K, van Roy F: Nuclear localization of the p120(ctn) Armadillo-like catenin is counteracted by a nuclear export signal and by E-cadherin expression. Proc Natl Acad Sci U S A 1999, 96:7980-7985

41. Kelly KF, Otchere AA, Graham M, Daniel JM: Nuclear import of the BTB/POZ transcriptional regulator Kaiso. J Cell Sci 2004, 117: $6143-6152$

42. Dai SD, Wang Y, Jiang GY, Zhang PX, Dong XJ, Wei Q, Xu HT, Li QC, Zhao C, Wang EH: Kaiso is expressed in lung cancer: its expression and localization is affected by p120ctn. Lung Cancer 2010, 67:205-215

43. Chang WC, Lee YC, Liu CL, Hsu JD, Wang HC, Chen CC, Wang CJ: Increased expression of iNOS and c-fos via regulation of protein tyrosine phosphorylation and MEK1/ERK2 proteins in terminal bronchiole lesions in the lungs of rats exposed to cigarette smoke. Arch Toxicol 2001, 75:28-35

44. Zhang Q, Adiseshaiah P, Reddy SP: Matrix metalloproteinase/epidermal growth factor receptor/mitogen-activated protein kinase signaling regulate fra-1 induction by cigarette smoke in lung epithelial cells. Am J Respir Cell Mol Biol 2005, 32: $72-81$

45. Volm M, Drings P, Wodrich W: Prognostic significance of the expression of c-fos, c-jun and c-erbB-1 oncogene products in human squamous cell lung carcinomas. J Cancer Res Clin Oncol 1993, 119: 507-510

46. Subramanian J, Govindan R: Lung cancer in never smokers: a review. J Clin Oncol 2007, 25:561-570 